\title{
Monitoring Needs and Goal-directed Fluid Therapy Within an Enhanced Recovery Program
}

Gary Minto, MBChB, FRCA ${ }^{a, *}$, Michael J. Scott, MB ChB, MRCP, FRCA, FFICM ${ }^{b}$, Timothy E. Miller, MB ChB, FRCA ${ }^{\mathrm{C}}$

\section{KEYWORDS}

- Fluid therapy • Colorectal surgery $\bullet$ Cardiac output monitoring $\bullet$ Enhanced recovery

\section{KEY POINTS}

- Enhanced recovery principles mitigate against many of the factors that traditionally led to relative hypovolemia in the perioperative period.

- Individualization of fluid prescription requires consideration of clinical signs and hemodynamic variables.

- A large literature spanning 4 decades supports goal-directed fluid therapy.

- Application of this evidence to justify stroke volume optimization in the setting of major surgery within an enhanced recovery program is controversial.

Individuals having major abdominal surgery need perioperative fluid supplementation. This requirement is caused by:

- A physiologic stress response to the surgery. Incision and tissue handling trigger endocrine and inflammatory changes that lead to:

- Redistribution of water from body fluid compartments

- Endothelial changes promoting leakage of fluid out of capillaries

- Redistribution of blood flow

- Activation of sodium and water retention mechanisms

- The magnitude of the stress response varies between individuals, and even within the same individual, depending on the condition in which they present

Conflicts of interest: G. Minto declares he has no conflicts of interest.

${ }^{a}$ Department of Anaesthesia \& Perioperative Medicine, Plymouth Hospitals NHS Trust, Plymouth University Peninsula School of Medicine, Plymouth PL6 8DH, UK; ${ }^{b}$ Department of Anaesthesia and Intensive Care Medicine, Royal Surrey County Hospital, University of Surrey, Guildford GU1 7XX, UK; ' Department of Anesthesiology, Duke University Medical Center, Durham, NC 27710, USA

* Corresponding author.

E-mail address: gary.minto@nhs.net 
for surgery. Patients who are acutely ill tend to have a greater inflammatory response; this may be modified by preoperative factors such as nutritional status, neoadjuvant chemotherapy and radiotherapy, antibiotics, and steroids.

- Replacement of losses:

- Preoperative dehydration if oral fluids are withheld

- These effects may be magnified by bowel preparation

- Losses caused by the underlying disorder (eg, preoperative vomiting, diarrhea, or evaporative losses)

- Blood loss, although major hemorrhage is rare during elective bowel surgery

- Hemodynamic changes induced by anesthesia:

- Vasoparesis and venodilatation in response to neuraxial blockade ${ }^{1}$

- Drug effects on vasomotor tone and cardiac contractility; in general, anesthesia promotes vasodilatation

- Vasoconstriction in response to pressor agents

- Hemodynamic changes induced by surgical conditions:

- Positioning (eg, head up or down, prone ${ }^{2}$ )

- Pneumoperitoneum to facilitate laparoscopic surgery

- Reduced oral intake in the postoperative period, which may be complicated by intestinal ileus, excessive use of opioids, gastric tubes, nausea, vomiting.

Many of these mechanisms promote hypovolemia, which is a deficit in intravascular fluid volume.

\section{PERIOPERATIVE FLUID REQUIREMENTS IN THE CONTEXT OF ENHANCED RECOVERY}

In contrast with the emergency setting, in which many of the pathophysiologic perturbations discussed earlier are already in place at the time of surgery, enhanced recovery mitigates against most of these factors.

- Patients are brought to theater in a well-hydrated state, having also been provided with preoperative carbohydrate drinks.

- Routine bowel preparation is avoided for most colonic resections.

- Laparoscopic or small-incision surgery seeks to minimize physiologic disturbance.

- Long-acting opioids, which may cause ileus, are avoided.

- In many enhanced recovery (ER) programs, epidural blockade is also avoided, minimizing hemodynamic changes caused by regional anesthesia. ${ }^{3}$

- ERs incorporate a general fluid therapy philosophy of avoidance of sodium and water overload (discussed later).

- Early resumption of enteral feeding, early mobilization, and reduced tubes and drains are intended to allow patients to self-regulate their fluid and nutritional intake from soon after surgery. ${ }^{4}$

For these reasons perioperative fluid management may be different within ER pathways than in other settings.

\section{GENERAL APPROACHES TO INTRAOPERATIVE FLUID THERAPY Fixed-volume Strategies}

During surgery, intravenous fluid administration is necessary. In the past, a simplistic fixed-volume approach has been used, whereby an estimated baseline fluid regimen is commenced, and then modified based on measurement of preoperative and ongoing losses, and on information from conventional hemodynamic monitoring. 
Whether this baseline fluid regimen should be liberal or restrictive has been the source of much debate. A key factor here is so-called third-space loss, which is the concept that a large amount of fluid (perhaps $10 \mathrm{~mL} / \mathrm{kg} / \mathrm{h}$ ) shifts from the intravascular into the peritoneal space and evaporates when the abdomen is open during surgery, and that a degree of fluid redistribution and capillary leak persists for a time after surgery, creating a risk of relative hypovolemia. A liberal intraoperative fluid regimen is variably defined, but typically involves a bolus of 500 to $1000 \mathrm{~mL}$ of intravenous crystalloid before the commencement of surgery, around $10 \mathrm{~mL} / \mathrm{kg} / \mathrm{h}$ during surgery, and a further 2 to $3 \mathrm{~L}$ intravenous fluid after surgery, totaling more than $5 \mathrm{~L}$ in the first postoperative day. ${ }^{5}$

Although deeply entrenched in clinical teaching and practice for decades, there is minimal evidence that third-space loss occurs. ${ }^{6}$

There is a paucity of large-scale perioperative studies to date, but many small trials, systematic reviews, and meta-analyses consistently favor a restrictive approach. ${ }^{5,7-9}$ It is relevant that a recent randomized controlled trial (RCT) of resuscitation strategies for gastroenteritis in sub-Saharan African children linked liberal fluid prescription with an increased mortality. ${ }^{10}$

What constitutes restrictive is also variably defined; avoidance of fluid excess is perhaps a more appropriate terminology. ${ }^{11}$ The summary from meta-analysis is that patients in the restrictive arms of studies received around $1500 \mathrm{~mL}$ less intraoperative crystalloid (95\% confidence interval [Cl], 986-2154 mL) than those in the liberal group ${ }^{5}$ and that this was associated with a lower rate of complications and a shorter hospital stay.

A problem with fixed-volume strategies is that they disregard interindividual variation. The ideal is to individualize fluid therapy so as to avoid harmful effects of either hypovolemia or fluid overload (Fig. 1).

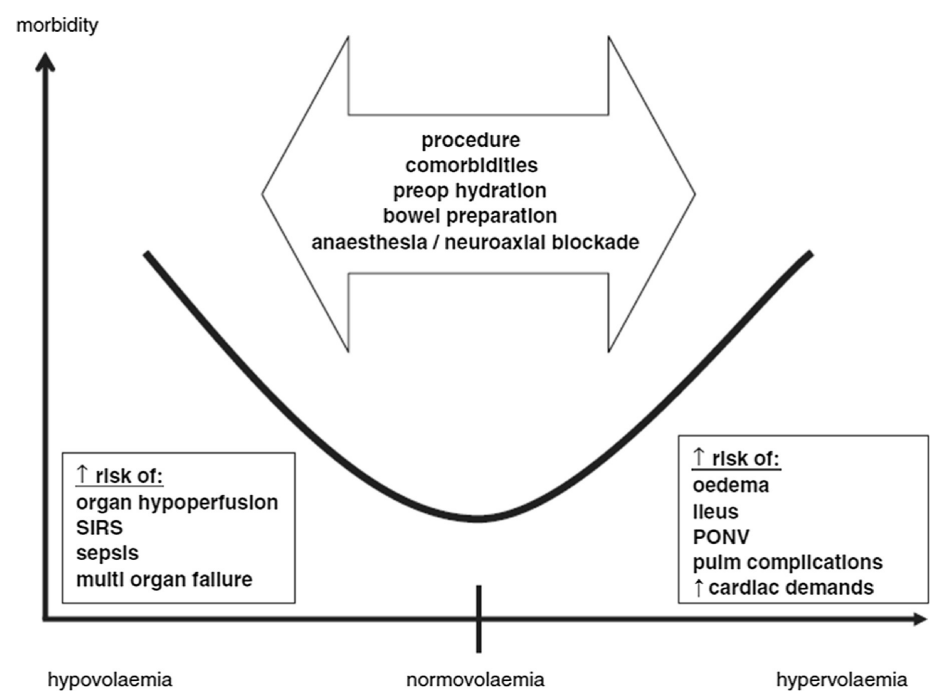

Fig. 1. A conceptual curve portraying the relationship between perioperative administered fluid volume and postoperative morbidity, and factors influencing shift of the curve (arrow). Boxes indicate the risk of complications associated with deviation from normovolemia. PONV, postoperative nausea and vomiting; SIRS, systemic inflammatory response syndrome. (From Bundgaard-Nielsen M, Secher N, Kehlet H. 'Liberal' vs. 'restrictive' perioperative fluid therapy a critical assessment of the evidence. Acta Anaesthesiol Scand 2009;53:850; with permission.) 


\section{Conventional Hemodynamic Monitoring}

Individualization of fluid prescription needs to take into account gender, age, complicating illnesses, body composition, and preoperative hydration. ${ }^{12}$ In clinical practice, regimens are not fixed doses; fluid administration is modified at the discretion of attending clinicians, based on consideration of various clinical signs, hemodynamic variables, and biomarkers.

- Heart rate, systolic and diastolic blood pressure, and pulse pressure are realtime hemodynamic measurements that are readily available during and after surgery; however, these are not a good reflection of central blood volume, because they are also affected by anesthesia and surgical stress. Moreover, compensatory changes, particularly splanchnic vasoconstriction, can mask hypovolemia. In a healthy volunteer study, heart rate and BP remained stable despite venesection of $25 \%$ of blood volume. ${ }^{13}$

- Peripheral perfusion and temperature may similarly be influenced by other perioperative factors.

- Urine output is an inaccurate reflection of central blood volume: pressure diuresis may occur even if there is relative hypovolemia; conversely, the surgical stress response promotes water and salt retention, and pneumoperitoneum may cause oliguria.

- Central venous pressure measurements do not correlate well with intravascular volume because a multitude of factors (muscle relaxation, remifentanil infusions, epidural and spinal anesthesia, and pneumoperitoneum) affect venous tone. In general, central venous pressure does not predict fluid responsiveness (unless very low) so cannot be used to identify when patients need more fluid, ${ }^{14}$ although it may serve as an indicator of venous capacitance to avoid overinfusion.

- Biomarkers, such as arterial lactate, and a comparison of central venous and arterial oxygen saturations to characterize tissue oxygen extraction have some potential, ${ }^{15}$ but are not highly responsive to real-time hemodynamic changes. Global measurements may not reflect perfusion in particular tissues, especially the intestines, and algorithms incorporating their use are complex. The situation is further complicated because central venous sampling is from the superior vena cava and therefore a true mixed venous saturation is not obtained. Trending and response to fluid boluses may be useful in the immediate postoperative period (4-6 hours); however, in most surgical patients, perturbations in measured tissue oxygenation during surgery are minimal in contrast with the oxygen delivery changes seen in critically ill patients. ${ }^{16-18}$

\section{Advanced Hemodynamic Monitoring}

The ability to use extra hemodynamic information to judge fluid administration during surgery is an attractive concept. This technique was previously difficult because it generally required insertion of a pulmonary artery catheter and thermodilution techniques to measure cardiac stroke volume and other variables. However, minimally invasive technology is now readily available in the form of cardiac output or flowbased monitors, which seem to provide reasonably accurate estimations of functional circulating volume and can be used continuously throughout surgery to monitor for any deficit or excess. Prominent techniques are:

- Arterial waveform analysis; converting the pulse pressure signal into a nominal stroke volume 
- Esophageal Doppler; deriving stroke volume from blood flow in the descending thoracic aorta

- Plethysmography; in effect, using the fullness of the fingertip with each heartbeat to estimate circulating blood volume

- Finger cuffs; using the volume clamp method to continuously measure blood pressure and stroke volume from a finger cuff

- Partial carbon dioxide rebreathing; using the reverse Fick principle to calculate cardiac output

- Transthoracic bioimpedance and bioreactance; exploiting the variation in electrical resistance with intrathoracic blood volume during the cardiac cycle

In practice, a monitor's ability to display a nominal cardiac output is of questionable value. To be of practical use for perioperative fluid therapy, measured variables need to be incorporated into a dynamic algorithm, so that the user can direct fluid therapy toward specific hemodynamic targets; so-called goal-directed fluid therapy (GDFT). Most perioperative algorithms are based on stroke volume optimization (SVO) (Box 1).

A perioperative fluid therapy strategy based on detection and treatment of occult hypovolemia with small fluid challenges should reduce adverse effects related to

\section{Box 1 \\ Cardiac output monitors in practice: the SVO concept}

Although the new generation of advanced hemodynamic monitors are commonly referred to as cardiac output monitors, their main clinical application is to characterize fluid responsiveness; that is, a measurable increase in stroke volume in response to a fluid challenge. Fluid responsiveness is taken as being synonymous with hypovolemia. It is thought that individuals are normovolemic when their stroke volume is at the shoulder of the Frank-Starling curve in the supine position. According to this model, patients are hypovolemic when the stroke volume is on the steep, ascending leftward part of the Frank-Starling curve, whereas an increase in preload does not translate to an increase in stroke volume when stroke volume is on the plateau. It is possible to exploit this clinically with a fluid challenge: a minimal response suggests that stroke volume is on the plateau. If the stroke volume increases $10 \%$ or more within 5 minutes, then the patient is assumed be on the steep upward part of the curve and to have been fluid responsive (as opposed to no increase, which would place the patient on the flat normovolemic portion of the curve).

Most stroke volume monitors use this concept in their suggested algorithms for fluid therapy. Some monitors in addition are able to continuously display so-called dynamic flow indicators: stroke volume variability, pulse pressure variation, and systolic pressure variability, all derived from the arterial pressure waveform. These indicators are essentially mini fluid challenges provided by swings in intrathoracic pressure induced by intermittent positive pressure mechanical ventilation. The stroke volume (or pulse pressure or systolic pressure) response during each respiratory cycle is inversely related to circulating volume. When the stroke volume is on the plateau of the Starling curve, arterial pressure swings are minimized; when hypovolemia is present, variability increases. Certain conditions are essential when using dynamic flow indices: a regular pulse (so that variability in diastolic filling time is not a factor), a sufficiently large tidal volume to cause a swing in intrathoracic pressure with mechanical ventilation, and an absence of spontaneous breathing efforts (which would interfere with intrathoracic pressure) (Fig. 2).

In clinical practice, the SVO algorithm is used, and other information, such as conventional hemodynamic signals to maintain the ideal stroke volume, is also considered. In effect, the additional advanced monitoring gives the user confidence to administer or withhold fluid at certain stages of the operation at which they otherwise might not (ie, bespoke individualized fluid therapy).

Intraoperative conditions such as pain, position, and pneumoperitoneum may have an effect on stroke volume (or stroke volume variation) at a particular moment, but the ultimate aim is to have each patient euvolemic at the end of surgery. 


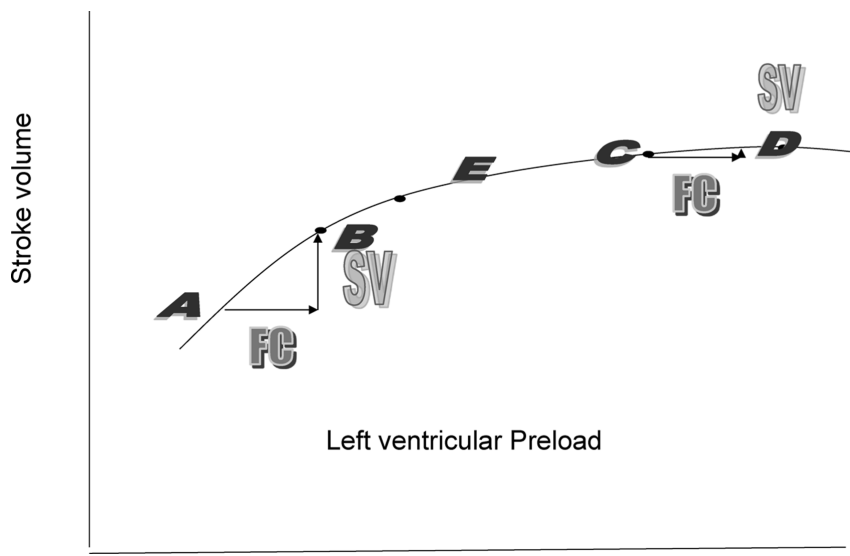

Fig. 2. Response of stroke volume (SV) to a fluid challenge (FC) is a marker of position on the Frank-Starling curve. Where SV increases more than $10 \%$ in response to $200 \mathrm{~mL}$ of isotonic fluid $(A$ to $B)$, patients are described as fluid responsive. Where SV does not increase to this extent ( $C$ to $D$ ), then no further FC is given. In the case of SV variation and other dynamic flow indices, the SV (or other variable such as systolic pressure) varies in response to preload changes induced by mechanical ventilation rather than an FC per se, but the concept is similar. Where SV variation is greater than $12 \%$ patients are likely to be fluid responsive, and where SV variation is less than $10 \%$ they are not likely to be. SVO refers to using fluid (either colloid or isotonic crystalloid) to keep the SV at the shoulder of the curve $(E)$, which is similar to keeping SV variation less than $10 \%$.

inadequate circulating volume, and, overall, this is what the literature shows. Compared with a fixed-dose fluid regimen modified by conventional static measurements, GDFT is associated with better clinical outcomes.

The definitive 2013 Cochrane Systematic Review on the subject, "Perioperative Increase in Global Blood Flow to Explicit Defined Goals and Outcomes after Surgery," includes 31 studies comprising 5292 participants and shows a clear reduction in complications, particularly renal and respiratory failure and wound infections. ${ }^{19}$

There is also biological plausibility. In early studies, patients in the GDFT group had significantly higher end-operative stroke volume ${ }^{20-23}$ compared with conventional practice, suggesting that occult hypovolemia was being avoided. The amounts of fluid were not very different; the GDFT group on average received a larger amount of intraoperative colloid $(467 \mathrm{~mL}$; $95 \% \mathrm{Cl}, 331-603)$ than the non-goal-directed group. ${ }^{5}$ The hypothesis is that the effect is caused by timing; not only is tissue ischemia avoided but patients are also saved from fluid loading at inappropriate times. Excess fluid given at the wrong time is not neutral, it results in edema ${ }^{24}$ and can damage the endothelial glycocalyx, ${ }^{25}$ promoting further edema.

In 2011, from the available evidence, the UK National Institute for Healthcare and Clinical Excellence strongly endorsed cardiac output monitoring to guide perioperative fluid administration in appropriate cases, ${ }^{26}$ and GDFT has been enthusiastically adopted as a pillar of enhanced recovery care. ${ }^{27}$

\section{TERMINOLOGY: GOAL DIRECTED OR GOAL MISDIRECTED?}

On a cautionary note, although systematic reviews of the literature may consistently support goal-directed therapy (GDT), ${ }^{19,28-31}$ all of these meta-analyses combine evidence from many settings. GDT is a vague term, meaning different things to different 
people and, depending on the clinical environment, sometimes even different things to the same person. ${ }^{11}$ It can refer to perioperative fluid management, clinicians driving oxygen delivery to supramaximal levels in critical care patients, or early treatment of sepsis in the emergency department.

Even when considered just in surgical patients, fluid therapy can be delivered with or without inotropes, preoperatively, intraoperatively, and postoperatively, and to patients who may or may not be critically ill. GDFT, in whatever guise, is designed to optimize global tissue oxygen delivery and oxygen consumption. For most elective perioperative patients it seems that this can be achieved by optimization of preload alone (ie, provision of fluid).

Therefore when interpreting the extensive available literature on GDFT, it is important to appreciate the distinction between these 3 clinical questions:

- In high-risk patients, do interventions to increase global oxygen delivery during the perioperative period produce clinical benefit?

- For all patients having major surgery, is the use of additional monitoring to measure fluid responsiveness beneficial? - Does SVO work?

- Is it necessary to optimize patients all the way through surgery or does a period of postoperative optimization of oxygen delivery and reperfusion restore oxygen, nutrients, and blood pressure after a period of cellular injury?

Perhaps clinicians should be cautious in extrapolating evidence from one setting to the other. Stroke volume is a surrogate; in the theater environment, it is hoped that hemodynamic interventions will optimize oxygen delivery but there is currently no useful monitor of tissue well-being to verify that interventions are working in real time.

\section{GOAL-DIRECTED FLUID THERAPY SPECIFICALLY IN THE ENHANCED RECOVERY SETTING}

The ER is viewed as a package to be applied, bringing such an improvement in quality of care that there is little need to unpick the particular element of the package that affords the most benefits. There is a clear association between compliance with all the elements and a better clinical outcome. ${ }^{32}$ Perioperative GDFT is such a cornerstone of enhanced recovery that the 20 or so steps that constitute recognizable ER programs have been simplified into a trimodal system of:

- GDFT

- Good analgesia

- Everything else els $^{33}$

GDFT is successfully incorporated into 23-hour-stay laparoscopic colorectal ${ }^{34}$ and short-stay liver resection pathways. ${ }^{35}$ Even this evidence is conflicting because patients in colorectal surgery were stroke volume optimized throughout, whereas patients having liver resection were rendered hypovolemic with low-pulse-pressure anesthesia during surgery and resuscitated to restore stroke volume as rapidly as possible after the end of the liver resection. This raises the question of whether there are key periods to aim for SVO and whether it is necessary throughout the operative period. It may be more beneficial to reduce stroke volume and blood pressure to reduce blood loss as long as hypoperfusion is limited and parameters are restored at the end of surgery.

So much of the evidence for GDFT is from an era when perioperative surgical practice was different; it is probable that many control patients in those trials were hypovolemic during surgery. As overall perioperative care changes, so apparently does the measurable impact of any particular intervention, including a complex intervention like 
GDFT. ${ }^{28}$ If clinicians confine their analysis to RCTs of GDFT versus conventional management within a defined ER program, it is apparent that there is a paucity of them. A systematic review published in 2014 found only 4 in elective colorectal surgery. ${ }^{36-40}$ Two of these ${ }^{37,38}$ were not included in the Cochrane Review of perioperative increase in global blood flow to the explicit defined goals cited earlier. The largest multicentre study of perioperative GDFT to date, OPTIMISE, contains a meta-analysis of all relevant trials up to early 2014, including those two, and indicates an overall benefit for GDFT, but the overall result is still influenced by all the early studies. ${ }^{31}$

Current evidence suggests no effect of SVO guided by esophageal Doppler monitoring on complication rates (Table 1) or length of stay after colorectal surgery conducted within ER pathways.

This lack of effect may be a result of newer trials being conducted within an environment of optimized perioperative care. As a result, patients are more likely to be fluid replete during surgery and may not have benefitted from targeted fluid administration (Fig. 3). Even among these 4 trials, there is some statistical heterogeneity, reflecting different conditions of the included studies. In 2 of the RCTs, ${ }^{37,38}$ GDFT was tested against fluid restriction, whereby meticulous attention was paid to fluid balance. $\mathrm{Pa-}$ tients came to theater in a euvolemic state, a conservative baseline infusion of intraoperative crystalloid was advocated, intraoperative losses were carefully monitored and replaced volume for volume with colloid, and response was monitored with conventional hemodynamic signals. Around two-thirds of the participants in 1 study had laparoscopic surgery, minimizing blood loss and physiologic fluid shifts. ${ }^{38}$ An average of 900 to $1300 \mathrm{~mL}$ of crystalloid and 300 to $500 \mathrm{~mL}$ of colloid were given during surgery lasting around 2 hours. Esophageal Doppler-guided GDFT had no benefit compared with this regimen in either study.

In contrast, in a third study, although perioperative care was provided within an ER pathway, control patients had a considerably more liberal perioperative fluid regimen. ${ }^{39}$ In addition, intervention patients were given an additional $1.3 \mathrm{~L}$ of intraoperative colloid by an investigator; apparently to their detriment because they remained in hospital for 2 days longer than controls. Our interpretation is that the GDFT algorithm that was used failed to guard against fluid overload in patients who were receiving a liberal baseline fluid regimen (discussed later).

\section{Table 1}

Forest plot comparing overall complication rates in esophageal Doppler monitor (ODM) and control groups after colorectal surgery within an enhanced recovery protocol. A MantelHaenszel random-effects model was used for meta-analysis. Odds ratios are shown with $95 \%$ confidence intervals

\begin{tabular}{|c|c|c|c|c|c|c|}
\hline \multirow[b]{2}{*}{ References } & \multicolumn{2}{|c|}{ Complications } & \multirow{2}{*}{$\begin{array}{l}\text { Weight } \\
(\%)\end{array}$} & \multirow[b]{2}{*}{ Odds Ratio } & \multirow[b]{2}{*}{ Odds Ratio } & \\
\hline & ODM & Restriction & & & & \\
\hline Brandstrup et $a^{38}$ & 23 of 71 & 24 of 79 & 37.5 & $1.10(0.55,2.19)$ & & $\rightarrow-$ \\
\hline Challand et $\mathrm{al}^{39}$ & 63 of 89 & 60 of 90 & 44.6 & $1.21(0.64,2.28)$ & & - \\
\hline$\underline{\text { Srinivasa et } \mathrm{al}^{37}}$ & 26 of 37 & 26 of 37 & 18.0 & $1.00(0.37,2.71)$ & & \\
\hline \multirow[t]{2}{*}{ Total } & 112 of 197 & 110 of 206 & 100.0 & $1.13(0.74,1.72)$ & & \\
\hline & & & & & $\begin{array}{ccc}1 & 1 & 1 \\
0.1 & 0.2 & 0.5 \\
& \text { Favours } & \text { ODM }\end{array}$ & $\begin{array}{llll}1 & 1 & 1 & 10\end{array}$ \\
\hline
\end{tabular}

Heterogeneity: $\tau^{2}=0.00 ; \chi^{2}=0.11,2$ degrees of freedom, $P=.95 ; I^{2}=0 \%$.

Test for overall effect: $Z=0.56, P=.58$.

From Srinivasa $S$, Lemanu $D$, Singh $P$, et al. Systematic review and meta-analysis of oesophageal Doppler-guided fluid management in colorectal surgery. Br J Surg 2013;100:1705; with permission. 


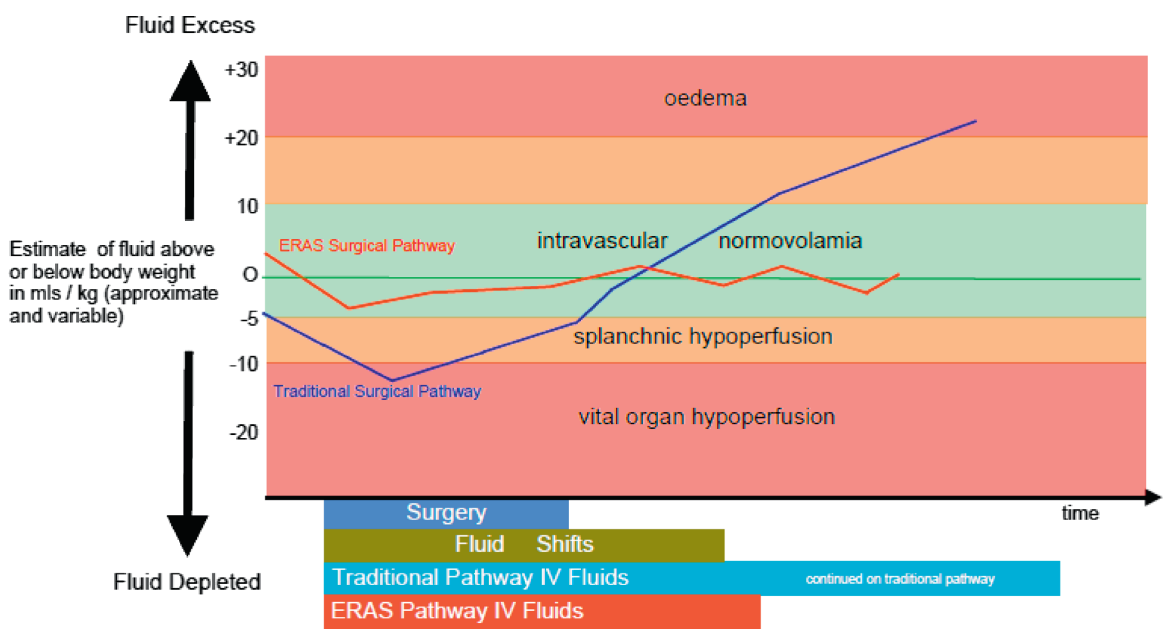

Fig. 3. Conceptual model contrasting perioperative fluid status changes and fluid therapy in the settings of enhanced recovery after surgery (ERAS) and traditional surgery. Surgery and physiologic shifts tend to reduce intravascular volume during the early stages of the perioperative period, whereas intravenous (IV) fluid may promote a relative fluid excess in the hours to days afterward. In general, ERAS minimizes these physiologic changes.

Perioperative fluid therapy may be restrictive, liberal, goal directed, or a combination thereof. Overall, there is evidence that, within an ER setting, a carefully monitored restrictive regimen is equivalent to intraoperative GDFT for patients having elective colorectal surgery, and that both of these are better than liberal fluid therapy supplemented by SVO.

Note that, in a small study of 32 patients having 2-hour colonic resections within a multimodal ER regimen, a regimen of restrictive fluids (median $1640 \mathrm{~mL}$ of intravenous fluid on the day of surgery) compared with liberal fluids (median $5050 \mathrm{~mL}$ ) was associated with more complications (18 in 6 patients vs 1), ${ }^{41}$ suggesting that it is possible to be overly restrictive. In contrast, in a study examining compliance with components of ER, avoidance of fluid overload was the most important factor in avoiding complications: for every liter of fluid administered, postoperative complications (mainly cardiorespiratory) increased by $32 \% .^{32}$ Every liter of postoperative fluid excess is associated with an additional day in hospital stay. ${ }^{3}$ For colorectal surgery within ER, euvolemia is desirable, but the best way to achieve this has not been resolved.

In other major abdominal surgical settings evidence is more limited. A study of esophageal Doppler monitor (ODM)-guided GDFT in cystectomy ${ }^{42}$ suggests benefit, although the study is not specified as being within ER, and an RCT of GDFT for major gynecology ${ }^{43}$ showed no difference in complication rates, although it had an overall length of stay of 11 days. Single studies constitute insufficient evidence to make conclusions.

\section{CONTROVERSIES: VALIDITY OF THE STROKE VOLUME OPTIMIZATION CONCEPT}

A valid question is whether novel hemodynamic devices and indices provide a false sense of security. As discussed, the monitors are claimed to be effective to characterize fluid responsiveness, which is taken as a sign of hypovolemia (see Box 1). A 
problem with this approach is that the scientific foundations are not well established. Guidance that clinicians should give $200 \mathrm{~mL}$ of fluid (until recently colloid was advocated) and wait 5 minutes for a 10\% increase in stroke volume seems arbitrary.

The association of dynamic flow indices with fluid responsiveness has a more sound methodological basis. A receiver operator curve comparing the variable in question (eg, stroke volume variation) with a gold standard method of assessing volume status (eg, LV volume measured by transesophageal echo) is used to establish thresholds for prediction of fluid responsiveness. ${ }^{44} \mathrm{~A}$ further degree of sophistication is introduced by identifying 2 thresholds; one that prioritizes specificity (not giving fluid to patients who do not need it) and the other that prioritizes sensitivity (ie, making sure that all cases of hypovolemia are detected). The interval between them is a zone of uncertainty (clinicians cannot be certain whether patients are- fluid responsive or not) in which around $25 \%$ of readings from typical perioperative cohorts occur. ${ }^{45}$

Sophisticated as they may be, clear evidence of benefit using dynamic flow indices to guide GDFT during contemporary surgery is scarce. The variables require certain conditions: a delivered tidal volume more than $7 \mathrm{~mL} / \mathrm{kg}$, absence of spontaneous breathing activity, absence of cardiac rhythm abnormalities (which make diastolic filling inconsistent), and ideally a responsive breath-by-breath calculation method, many of which are lacking in a high proportion of intraoperative patients. ${ }^{46}$ The possibility exists that an open abdomen attenuates pressure swings, whereas a pneumoperitoneum accentuates it.

Moreover, comparisons of monitors to predict fluid responsiveness in the same patients often do not agree closely with one another. ${ }^{47}$ In addition, even the assertion that fluid responsiveness indicates hypovolemia is questionable. In a recent study around half of the well-hydrated volunteer subjects showed a stroke volume increase of more than $10 \%$ in response to a passive leg raise (in effect, a fluid challenge). ${ }^{48}$

Further, in contrast with the more sequentially evolving environment of critical care, the intraoperative setting is characterized by rapid changes in stimuli, endogenous catecholamine levels, effects of drugs, and hemorrhage, all of which may affect stroke volume. Rather than having a single Frank-Starling curve, depending on these factors, individuals move serially between a family of ventricular function curves (depending on cardiac contractility and afterload), so it is difficult to be sure what the optimum stroke volume is at a particular moment. ${ }^{49}$

It has been implied with SVO that advanced hemodynamic monitoring protects against a fluid overload, but perhaps this is not the case. The distinction between SVO and stroke volume maximization is more than semantic ${ }^{50}$ : any excess fluid could be detrimental. ${ }^{24}$ As well as signals about relative hypovolemia, GDFT algorithms require clear stopping thresholds (ie, signs that the circulating volume is full) and it is possible that this aspect of the algorithm has been found wanting when SVO has been tested under ER conditions.

\section{REQUIRED RESEARCH}

\section{Effectiveness Trial Within an Enhanced Recovery Environment}

Translation of a complex intervention into clinical benefit requires that the intervention is shown to work when clinicians apply it to patients in routine hospital practice. There are as yet no large RCTs investigating the clinical effectiveness of GDFT to improve outcomes within an ER context. It seems unlikely that additional hemodynamic information can lead to harm ${ }^{39}$ but, in a cost-constrained environment, in order to be costeffective GDFT has to be shown to be better. 
Adequate blinding of GDFT is difficult, so several trials are biased in that perioperative clinicians knew which group patients were allocated to, ${ }^{21,23,40}$ which may systematically affect their overall care or the determination of outcomes. In contrast, attempts to blind delivery of the intervention ${ }^{22,37-39}$ render the study protocol different from real-life use.

Any literature that comprises many single-center efficacy studies is open to the possibility of publication bias (ie, that only studies with a positive result are published); the Cochrane meta-analysis suggests that this is the case with the GDFT literature. ${ }^{19}$

Definitive sufficiently powered effectiveness trials are difficult to conduct: they require a cooperative network of centers capable of delivering an intervention (in this case GDFT) consistently within an ER program that is broadly similar across all the centers. Within an effectiveness trial, prospectively defined subgroup analysis (eg, types of surgery, levels of comorbidity, or aerobic fitness) may be analyzed to identify particular settings in which GDFT may be useful. ${ }^{51}$ An RCT of restrictive versus liberal fluid therapy for contemporary major abdominal surgery intending to recruit more than 2800 patients is underway ${ }^{52}$ in which there is a planned statistical interaction analysis to investigate the effect of GDFT superimposed on the basal fluid regimens, although this is no substitute for a prospective large head-to-head trial.

\section{DO PERIOPERATIVE INOTROPES CONVEY ADDITIONAL BENEFIT?}

A large proportion of trials included in meta-analyses allow for the use of inotropes as well as fluid challenges. OPTIMISE, the largest perioperative GDFT study to date, showed a strong but nonsignificant trend toward benefit for an arterial wave formderived SVO algorithm in 730 surgical patients; however, the intervention group in addition received a fixed-dose regimen of dopexamine during surgery and for 6 hours afterward. ${ }^{31}$ Positive outcomes may be caused by beta-agonist drugs having beneficial antiinflammatory and other metabolic effects. ${ }^{53} \mathrm{~A}$ much larger multicentre RCT investigating this premise further is currently being set up by the OPTIMISE investigating group, but this will take at least 5 years to produce answers. Another interpretation of the results is that it is 6 postoperative hours of close attention that pays dividends. This interpretation has echoes in the evolution of the literature ${ }^{17}$ on early GDT in patients presenting to the emergency department with septic shock. In a single-center efficacy trial, dramatic benefits were shown for GDFT compared with usual care. The protocol-based care for early septic shock (ProCESS) study recently tested the same GDFT regimen against 2 other fluid therapy strategies applied for 6 hours in 1341 patients across 31 centers and showed no difference in 60-day mortality from septic shock. ${ }^{54}$ It may be that what affects clinical outcomes is care being closely applied and monitored by diligent personnel, rather than monitors and algorithms per se.

ER focuses on simple steps of care delivered consistently and meticulously.

\section{SUBGROUPS OF BENEFIT?}

Laparoscopic colorectal surgery, involving a long period of pneumoperitoneum in a head-down position, has been described as minimally invasive surgery with maximal cardiovascular stress. This hemodynamic model of cardiac failure is in contrast with the compensated hypovolemia model of supine open abdominal surgery. Several observational and quality-improvement studies have been published by the Guildford group, for example, describing a $\mathrm{Do}_{2}$ target of $400 \mathrm{~mL} / \mathrm{min}-1 / \mathrm{m}-2$ to be a useful threshold in reducing complications, ${ }^{55}$ and several prominent GDFT studies include a large proportion of laparoscopic patients, ${ }^{38-40}$ with some attempt to adapt a generic 
SVO algorithm during pneumoperitoneum. ${ }^{38}$ However, prospective subgroup analysis (or ideally a GDFT RCT with a bespoke algorithm confined to laparoscopic resection patients) is lacking.

Some patients, by dint of comorbidities, underlying functional capacity, neoadjuvant chemoradiotherapy, or magnitude of surgical resection, are at higher risk than others of complications, but possible differential effects of GDFT in subgroups have not been adequately investigated in studies to date.

\section{CURRENT BEST PRACTICE}

Meticulous adherence to perioperative fluid administration is effective. ${ }^{27}$

GDT does not seem to cause harm (if done correctly) and although there is no evidence it seems sensible to match monitoring to surgical and patient risk.

Current consensus is that, for patients who arrive in theater in a euvolemic state, a crystalloid infusion of around $1.5 \mathrm{~mL} / \mathrm{kg} / \mathrm{h}$ during surgery is a reasonable baseline; additional fluid challenges can be used in response to measured losses and hemodynamic signals. It is perhaps easier to achieve individualized fluid therapy with a stroke volume-targeted approach guided by an advanced hemodynamic monitor, as long as particular attention is paid to stopping thresholds so as to avoid fluid excess. Because of a lack of specific evidence of benefit for GDFT when the physiologic disturbance attributable to the surgery is minimized, as in enhanced recovery, the point is not proved.

All fluid therapy and vasopressors should be administered according to the hemodynamic model at the particular stage of the operation; for example, steep head-up or head-down positions present different hemodynamic conditions. The aim is to have sufficient circulating volume and blood flow to avoid tissue hypoxia at all stages of the operation, and ultimately to have each patient euvolemic in the awake, supine state at the end of surgery, so that minimal postoperative intravenous fluid is required, and then only in response to clear clinical evidence of hypovolemia.

\section{REFERENCES}

1. Gould TH, Grace K, Thorne G, et al. Effect of thoracic epidural anaesthesia on colonic blood flow. Br J Anaesth 2002;89:446-51.

2. Biais $\mathrm{M}$, Bernard $\mathrm{O}, \mathrm{Ha} \mathrm{JC}$, et al. Abilities of pulse pressure variations and stroke volume variations to predict fluid responsiveness in prone position during scoliosis surgery. Br J Anaesth 2010;104(4):407-13.

3. Levy BF, Scott MJ, Fawcett WJ, et al. Randomized clinical trial of epidural, spinal or patient-controlled analgesia for patients undergoing laparoscopic colorectal surgery. Br J Surg 2011;98:1068-78.

4. Fearon KC, Ljungqvist $\mathrm{O}$, Von Meyenfeldt $\mathrm{M}$, et al. Enhanced recovery after surgery: a consensus review of clinical care for patients undergoing colonic resection. Clin Nutr 2005;24:466-77.

5. Corcoran T, Clarke S, Myles $P$, et al. Perioperative fluid management strategies in major surgery: a stratified meta-analysis. Anesth Analg 2012;114:640-51.

6. Brandstrup B, Svendsen C, Engquist A. Hemorrhage and operation cause a contraction of the extra cellular space needing replacement-evidence and implications? A systematic review. Surgery 2006;139:419-32.

7. Brandstrup B, Tønnesen H, Beier-Holgersen R, et al, Danish Study Group on Perioperative Fluid Therapy. Effects of intravenous fluid restriction on postoperative complications: comparison of two perioperative fluid regimens: a randomized assessor-blinded multicenter trial. Ann Surg 2003;238:641-8. 
8. Lobo DN, Bostock KA, Neal KR, et al. Effect of salt and water balance on recovery of gastrointestinal function after elective colonic resection: a randomised controlled trial. Lancet 2002;359:1812-8.

9. Nisanevich V, Felsenstein I, Almogy G, et al. Effect of intraoperative fluid management on outcome after intraabdominal surgery. Anesthesiology 2005;103:25-32.

10. Maitland K, Kiguli S, Opoka RO, et al, for FEAST Trial Group. Mortality after fluid bolus in African children with severe infection. N Engl J Med 2011;364(26):2483-95.

11. Roche A, Miller T. Goal-directed or goal-misdirected-how should we interpret the literature? Crit Care 2010;14:129.

12. Bundgaard-Nielsen M, Secher N, Kehlet H. 'Liberal' vs. 'restrictive' perioperative fluid therapy - a critical assessment of the evidence. Acta Anaesthesiol Scand 2009;53:843-51.

13. Hamilton-Davies C, Mythen M, Salmon J, et al. Comparison of commonly used clinical indicators of hypovolaemia with gastrointestinal tonometry. Intensive Care Med 1997;23:276-81.

14. Marik P, Baram M, Vahid B. Does central venous pressure predict fluid responsiveness? Chest 2008;134:172-8.

15. Donati A, Loggi S, Preiser JC, et al. Goal-directed intraoperative therapy reduces morbidity and length of hospital stay in high-risk surgical patients. Chest 2007; 132:1817-24.

16. Cohn S, Pearl R, Acosta S, et al. A prospective randomized pilot study of near-infrared spectroscopy-directed restricted fluid therapy versus standard fluid therapy in patients undergoing elective colorectal surgery. Am Surg 2010;76: 1384-92.

17. Rivers E, Nguyen B, Havstad S, et al, Early Goal-Directed Therapy Collaborative Group. Early goal-directed therapy in the treatment of severe sepsis and septic shock. N Engl J Med 2001;345(19):1368-77.

18. Shoemaker W, Appel P, Kram H, et al. Prospective trial of supranormal values of survivors as therapeutic goals in high-risk surgical patients. Chest 1988;94: 1176-86.

19. Grocott M, Dushianthan A, Hamilton M, et al, Optimisation Systematic Review Steering Group. Perioperative increase in global blood flow to explicit defined goals and outcomes following surgery. Cochrane Database Syst Rev 2012;(11):CD004082.

20. Conway DH, Mayall R, Abdul-Latif MS, et al. Randomised controlled trial investigating the influence of intravenous fluid titration using oesophageal Doppler monitoring during bowel surgery. Anaesthesia 2002;57:845-9.

21. Gan T, Soppitt A, Maroof M, et al. Goal-directed intraoperative fluid administration reduces length of hospital stay after major surgery. Anesthesiology 2002;97: 820-6.

22. Noblett SE, Snowden CP, Shenton BK, et al. Randomized clinical trial assessing the effect of Doppler-optimized fluid management on outcome after elective colorectal resection. Br J Surg 2006;93:1069-76.

23. Wakeling $\mathrm{H}$, McFall $\mathrm{M}$, Jenkins $\mathrm{C}$, et al. Intraoperative oesophageal Doppler guided fluid management shortens postoperative hospital stay after major bowel surgery. Br J Anaesth 2005;95:634-42.

24. Jacob M, Chappell D, Rehm M. Clinical update: perioperative fluid management. Lancet 2007;369:1984-6.

25. Woodcock TE, Woodcock TM. Revised Starling equation and the glycocalyx model of transvascular fluid exchange: an improved paradigm for prescribing intravenous fluid therapy. Br J Anaesth 2012;108(3):384-94. 
26. National Institute for Health and Clinical Excellence. CardioQ-ODM oesophageal Doppler monitor. Medical Technology Guide 3, March 2011. Available at: http:// www.nice.org.uk/nicemedia/live/13312/52624/52624.pdf. Accessed August 18, 2014.

27. Fawcett W, Mythen M, Scott M. Enhanced recovery: more than just reducing length of stay? Br J Anaesth 2012;109:671-4.

28. Hamilton MA, Cecconi M, Rhodes A. A systematic review and meta-analysis on the use of preemptive hemodynamic intervention to improve postoperative outcomes in moderate and high-risk surgical patients. Anesth Analg 2011;112:1392-402.

29. Gurgel ST, do Nascimento P. Maintaining tissue perfusion in high-risk surgical patients: a systematic review of randomized clinical trials. Anesth Analg 2011; 112:1384-91.

30. Lees N, Hamilton M, Rhodes A. Clinical review: goal-directed therapy in high risk surgical patients. Crit Care 2009;13:231.

31. Pearse RM, Harrison DA, MacDonald N, et al. Effect of a perioperative, cardiac output-guided hemodynamic therapy algorithm on outcomes following major gastrointestinal surgery: a randomized clinical trial and systematic review. JAMA 2014. http://dx.doi.org/10.1001/jama.2014.5305.

32. Gustafsson UO, Hausel J, Thorell A, et al. Adherence to enhanced recovery after surgery protocol and outcomes after colorectal cancer surgery. Arch Surg 2011; 46:571-7.

33. Mythen MG, Scott MJ. Anaesthetic considerations for enhanced recovery. In: Francis $\mathrm{N}$, Kennedy $\mathrm{RH}$, Ljungqvist $\mathrm{O}$, et al, editors. Manual of fast track recovery for colorectal surgery. London: Springer; 2012. p. 49-72.

34. Levy BF, Scott MJP, Fawcett WJ, et al. 23-hour stay laparoscopic colectomy. Dis Colon Rectum 2009;52:1239-43.

35. Jones C, Kelliher L, Dickinson M, et al. Randomized clinical trial on enhanced recovery versus standard care following open liver resection. Br J Surg 2013; 100:1015-24.

36. Srinivasa $S$, Lemanu $D$, Singh $P$, et al. Systematic review and meta-analysis of oesophageal Doppler-guided fluid management in colorectal surgery. $\mathrm{Br} J$ Surg 2013;100:1701-8.

37. Srinivasa S, Taylor M, Singh P, et al. Randomized clinical trial of goal- directed fluid therapy within an enhanced recovery protocol for elective colectomy. $\mathrm{Br} \mathrm{J}$ Surg 2012;100:66-74.

38. Brandstrup B, Svendsen P, Rasmussen M, et al. Which goal for fluid therapy during colorectal surgery is followed by the best outcome: near maximal stroke volume or zero fluid balance? Br J Anaesth 2012;109:191-9.

39. Challand C, Struthers R, Sneyd JR, et al. Randomized controlled trial of intraoperative goal-directed fluid therapy in aerobically fit and unfit patients having major colorectal surgery. Br J Anaesth 2012;108:53-62.

40. Senagore AJ, Emery T, Luchtefeld M, et al. Fluid management for laparoscopic colectomy: a prospective, randomized assessment of goal-directed administration of balanced salt solution or hetastarch coupled with an enhanced recovery program. Dis Colon Rectum 2009;52:1935-40.

41. Holte K, Foss N, Andersen J, et al. Liberal or restrictive fluid administration in fast-track colonic surgery: a randomized, double-blind study. $\mathrm{Br} J$ Anaesth 2007;99:500-8.

42. Pillai P, McEleavy I, Gaughan M, et al. A double-blind randomized controlled clinical trial to assess the effect of Doppler optimized intraoperative fluid management on outcome following radical cystectomy. J Urol 2011;186(6):2201-6. 
43. McKenny M, Conroy $\mathrm{P}$, Wong $\mathrm{A}$, et al. A randomised prospective trial of intraoperative oesophageal Doppler-guided fluid administration in major gynaecological surgery. Anaesthesia 2013;68:1224-31.

44. Michard F. Stroke volume variation: from applied physiology to improved outcomes. Crit Care Med 2011;39:402-3.

45. Cannesson M, Le Manach Y, Hofer CK, et al. Assessing the diagnostic accuracy of pulse pressure variations for the prediction of fluid responsiveness: a "gray zone" approach. Anesthesiology 2011;115:231-41.

46. Lansdorp B, Lemson J, van Putten $M$, et al. Dynamic indices do not predict volume responsiveness in routine clinical practice. Br J Anaesth 2012;108(3): 395-401.

47. Nordstrom J, Hallsjo-Sander C, Shore R, et al. Stroke volume optimization in elective bowel surgery: a comparison between pulse power wave analysis (LiDCOrapid) and oesophageal Doppler (CardioQ). Br J Anaesth 2013;110: 374-80.

48. Godfrey G, Dubrey S, Handy J. A prospective observational study of stroke volume responsiveness to passive leg raise in healthy non-starved volunteers as assessed by trans-thoracic echocardiography. Anaesthesia 2014;69:306-13.

49. Minto G, Struthers R. Stroke volume optimisation: is the fairy tale over? Anaesthesia 2014;69(4):291-6.

50. Bouwman RA, Boer C. Minimal invasive cardiac output monitoring: get the dose of fluid right. Br J Anaesth 2012;109(3):299-302.

51. Miller T, Roche A, Gan T. Poor adoption of hemodynamic optimization during major surgery: are we practicing substandard care? Anesth Analg 2011;112: 1274-6.

52. Myles PS, Bellomo R. A pivotal trial of fluid therapy for major abdominal surgery: need and equipoise. Crit Care Resusc 2011;13(4):278-80.

53. Bangash MN, Patel NS, Benetti E, et al. Dopexamine can attenuate the inflammatory response and protect against organ injury in the absence of significant effects on hemodynamics or regional microvascular flow. Crit Care 2013;17(2): R57. http://dx.doi.org/10.1186/cc12585.

54. ProCESS Investigators. A randomized trial of protocol-based care for early septic shock. N Engl J Med 2014;370(18):1683-93.

55. Levy BF, Fawcett WJ, Scott MJP, et al. Intra-operative oxygen delivery in infusion volume optimized patients undergoing laparoscopic colorectal surgery within an enhanced recovery programme: effect of different analgesic modalities. Colorectal Dis 2012;14:887-92. 